\title{
Снижение порога генерации с помощью легирования в лазерах среднего инфракрасного диапазона на основе $\mathrm{HgCdTe}$ с квантовыми ямами $\mathrm{HgTe}$
}

\author{
(C) А.А. Дубинов, В.Я. Алешкин, С.В. Морозов \\ Институт фозики микроструктур Российской академии наук, \\ 603950 Нижний Новгород, Россия \\ Нижегородский государственный университет им. Н.И. Лобачевского, \\ 603950 Нижний Новгород, Россия \\ E-mail: sanya@ipm.sci-nnov.ru
}

(Получена 30 января 2018 г. Принята к печати 20 фревраля 2018 г.)

\begin{abstract}
Предложена и проанализирована возможность значительного снижения порога межзонной генерации в лазерных структурах среднего инфракрасного диапазона на основе $\mathrm{HgCdTe}$ с квантовыми ямами $\mathrm{HgTe}$ с помощью легирования донорами путем введения $\delta$-слоев, расположенных вблизи квантовых ям. Показано, что при оптимальной поверхностной концентрации доноров в $\delta$-слое $4 \cdot 10^{10} \mathrm{~cm}^{-2}$ и рабочей температуре $>40 \mathrm{~K}$ возможно снижение порога лазерной генерации на длине волны 20 мкм более чем в 2 раза.
\end{abstract}

DOI: 10.21883/FTP.2018.09.46159.8833

\section{1. Введение}

В настоящее время для различных применений востребованы полупроводниковые лазеры, излучающие в среднем и дальнем инфракрасном (ИК) диапазоне длин волн. На сегодняшний день наиболее продвинутыми в этом диапазоне частот являются квантовые каскадные лазеры (ККЛ) на основе GaAs и InP [1]. Однако чрезвычайно сложная зонная схема каскадных структур, высокие требования по контролю параметров затрудняют их воспроизводимость. Кроме того, длина волны излучения в ККЛ сложно поддается перестройке.

Альтернативу ККЛ могут составить межзонные лазеры на основе полупроводников с узкой запрещенной зоной. Тройные полупроводниковые соединения, содержащие тяжелые элементы, в частности $\mathrm{HgCdTe}$, имеют ширину прямой запрещенной зоны, покрывающую широкий спектральный диапазон 0-1.6 эВ. Это дает возможность для наблюдения стимулированного излучения среднего ИК диапазона на межзонных оптических переходах при неравновесной накачке в таких полупроводниках. Недавние исследования оптических свойств волноводных структур $\mathrm{HgCdTe}$ с квантовыми ямами (КЯ) $\mathrm{HgTe,} \mathrm{которые} \mathrm{выращиваются} \mathrm{при} \mathrm{низких} \mathrm{темпе-}$ ратурах методом молекулярно-лучевой эпитаксии [2-4], выявили значительное повышение качества таких структур по сравнению с предшествующими, что позволило обнаружить стимулированное излучение (СИ) в среднем ИК диапазоне длин волн (вплоть до $\lambda \approx 20$ мкм) [5-9]. Однако пороговая интенсивность накачки для возбуждения наиболее длинноволнового СИ была довольно велика, что сказалось и на максимальной температуре $\left(T^{*}=45 \mathrm{~K}\right)$, при которой наблюдалось СИ. Одной из причин этого является существенная оже-рекомбинация в узкозонных КЯ НgТе.

В настоящей работе мы предлагаем и анализируем возможность значительного снижения порога генера- ции за счет выключения одной из возможных схем оже-рекомбинации с помощью легирования донорами за счет $\delta$-слоев, расположенных вблизи КЯ.

\section{2. Модель}

Оценку для пороговой плотности тока в лазере на основе $\mathrm{HgCdTe}$ с квантовыми ямами $\mathrm{HgTe}$, используя подход, развитый в работе [10] для лазера с объемной активной областью, можно получить из следующего выражения:

$$
j_{\text {th }}=q D\left(C_{n} \hat{n}^{2} \hat{p}+C_{p} \hat{p}^{2} \hat{n}+R_{d}+B \hat{n} \hat{p}\right),
$$

где $q$ - заряд электрона, $D$ - толщина волновода, $C_{n}$ и $C_{p}$ - коэффициенты оже-рекомбинации, $R_{d}=(\hat{n}+\hat{p}) / \tau$ и $\tau$ - скорость и время безызлучательной рекомбинации Шокли-Рида-Холла, $B$ - коэффициент излучательной рекомбинации, $\hat{n}$ и $\hat{p}-$ пороговые неравновесные объемные концентрации электронов и дырок соответственно. Для того чтобы перейти к случаю лазера с квантовыми ямами (двумерный случай) в качестве активной области, необходимо ввести поверхностные концентрации электронов и дырок, соответственно $n=Q d \hat{n}$ и $p=Q d \hat{p}$, где $Q$ и $d-$ число квантовых ям и их толщина. Для аналогичного лазера с оптической накачкой непосредственно в КЯ можно ввести связь между пороговой интенсивностью излучения оптической накачки $\left(I_{\mathrm{th}}\right)$ и пороговой плотностью тока $\left(j_{\mathrm{th}}\right)[11]$ :

$$
I_{\mathrm{th}}=\frac{W}{\eta q} j_{\mathrm{th}},
$$

где $W$ - энергия кванта излучения оптической накачки, $\eta$ - доля излучения оптической накачки, поглощенная в КЯ.

Для нахождения пороговых значений концентраций электронов и дырок можно использовать выражение для 
порога генерации лазера: $g(n, p)=\alpha$, где $g(n, p)-$ модовый коэффициент усиления активной среды с учетом внутренних потерь, а $\alpha$ - коэффициент потерь на выход излучения из лазера. Коэффициент $g(n, p)$ в случае, когда генерация излучения происходит на длине волны, соответствующей ширине запрещенной зоны $E_{g}$, определяется следующей формулой:

$$
g(n, p)=A\left(f_{n}-f_{p}\right),
$$

где $A=40 \mathrm{~cm}^{-1}$ (расчетное значение, полученное в работе [12] для лазерной структуры, аналогичной структуpe, в которой наблюдалось стимулированное излучение с $\lambda \approx 20$ мкм [8]),

$$
\begin{gathered}
f_{n}=\frac{1}{1+\exp \left\{\left(E_{g}-E_{\mathrm{F}}^{n}\right) / k_{\mathrm{B}} T\right\}}, \\
f_{p}=\frac{1}{1+\exp \left\{-E_{\mathrm{F}}^{p} / k_{\mathrm{B}} T\right\}}
\end{gathered}
$$

- функции распределения электронов и дырок для $\lambda=2 \pi \hbar c / E_{g}, c-$ скорость света в вакууме, $T-$ температура, $k_{\mathrm{B}}$ и $\hbar-$ постоянные Больцмана и Планка соответственно. Неравновесные квазиуровни Ферми для электронов $\left(E_{\mathrm{F}}^{n}\right)$ и дырок $\left(E_{\mathrm{F}}^{p}\right)$ в зависимости от концентраций полностью ионизованной донорной примеси $(N)$ в $\delta$-слоях и инжектируемых носителей ( $n$ и $p$ ) можно найти из следующих выражений в приближении чисто двумерного случая и параболических зон:

$$
\begin{gathered}
E_{\mathrm{F}}^{n}=E_{g}+k_{\mathrm{B}} T \ln \left[\exp \left\{\frac{\pi \hbar^{2}(n+N)}{k_{\mathrm{B}} T m_{n}}\right\}-1\right], \\
E_{\mathrm{F}}^{p}=-k_{\mathrm{B}} T \ln \left[\exp \left\{\frac{\pi \hbar^{2} p}{k_{\mathrm{B}} T m_{p}}\right\}-1\right],
\end{gathered}
$$

где $m_{n}$ и $m_{p}$ - массы плотности состояний электронов и дырок соответственно. Для достаточно узкозонных КЯ $\mathrm{HgTe}$ можно считать, что массы электронов и дырок в основных состояниях одинаковы: $m=m_{n} \approx m_{p}[8,12]$. В приближении $n=p$, учитывая формулы (3)-(5), можно записать для пороговой концентрации неравновесных носителей следующее выражение $(A>\alpha)$ :

$$
n=\frac{1}{P} \ln \left[\frac{1+\exp (-P N)}{1-\alpha / A}\right],
$$

где $P=\pi \hbar^{2} / m k_{\mathrm{B}} T$. Подставляя выражение (6) в формулы (1) и (2), можно найти соответственно значения пороговых плотности тока и интенсивности накачки в лазере.

Основным процессом в условиях интенсивной накачки при межзонной рекомбинации электрона и дырки в узкозонных полупроводниках является безызлучательная оже-рекомбинация. Известно, что причина увеличения скорости оже-рекомбинации лежит в увеличении разницы между эффективными массами электронов и дырок. Чем больше такая разница, тем ниже энергетический порог оже-рекомбинации. В конечном счете при очень большой разнице масс электронов и дырок результатом является практически беспороговый оже-процесс, когда при любой концентрации неравновесных носителей межзонная рекомбинация беспороговым образом происходит через безызлучательную оже-рекомбинацию. Обратно, в структурах с симметричным законом дисперсии порог оже-рекомбинации максимальный. Таким образом, даже в материале с очень малой щелью определенная симметрия законов дисперсии носителей позволяет подавить оже-рекомбинацию, которая является одним из важнейших факторов, ограничивающих возможность получения инверсии населенностей. Так, при симметричном дираковском законе дисперсии (с ненулевой массой носителей) оже-рекомбинация полностью запрещена в силу невозможности выполнения законов сохранения энергии и импульса при оже-процессе. В КЯ $\mathrm{HgTe} / \mathrm{HgCdTe}$ можно обеспечить аналогичную симметрию, причем подбор ширины КЯ позволяет добиться конечной энергетической щели, но в то же время сохранить симметричный закон дисперсии. Узкозонные КЯ НgТе обладают квази-дираковским, т. е. гиперболическим и симметричным, законом дисперсии для основных состояний электронов и дырок, по крайней мере в окрестности $k=0$. Как было показано в [8,9], это может существенно подавить оже-рекомбинацию между этими состояниями. Однако при продвижении генерации СИ в более длинные волны необходимо большее усиление для преодоления потерь Друде, которые увеличиваются квадратично с длиной волны. Это приводит к увеличению необходимой концентрации носителей и изменению функции распределения их в подзонах размерного квантования.

Если разность в энергиях первой и второй подзон электронов может в несколько раз превышать разность в энергиях первых подзон электронов и дырок (шири-

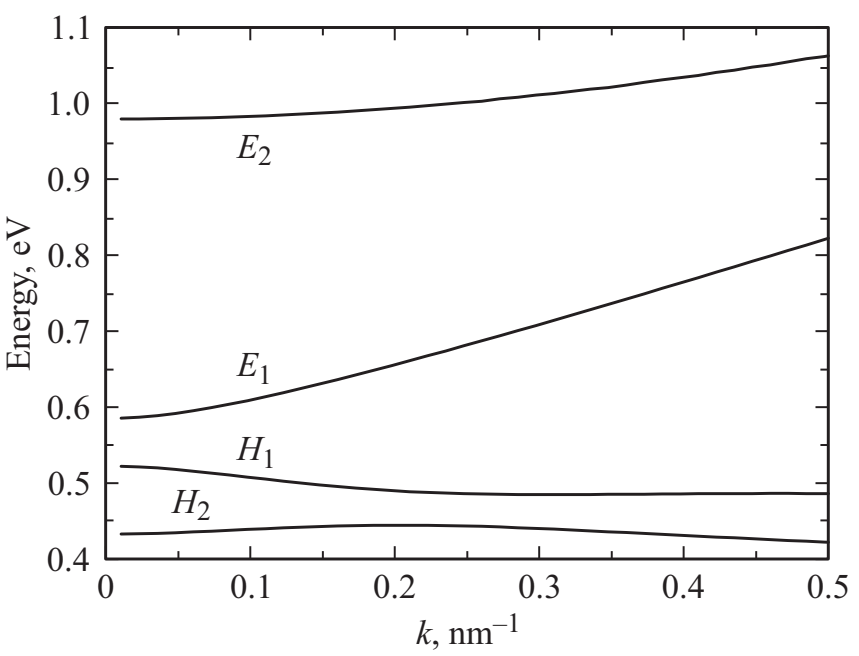

Рис. 1. Электронный спектр КЯ НgТе толщиной 4.6 нм, заключенной в барьеры $\mathrm{Hg}_{0.3} \mathrm{Cd}_{0.7} \mathrm{Te}(013)$, при $T=20 \mathrm{~K}$. Волновой вектор направлен по направлению [100]. $E_{1}$ и $E_{2}-$ 1-я и 2-я электронные подзоны соответственно; $H_{1}$ и $H_{2}-1$-я и 2-я дырочные подзоны соответственно. 
на запрещенной зоны в КЯ), то разность в энергиях первой и второй подзон дырок сопоставима с шириной запрещенной зоны. Кроме того, при больших значениях квазиимпульса закон дисперсии дырок перестает быть квази-дираковским. Это иллюстрирует рис. 1, на котором представлен электронный спектр КЯ HgТе толщиной 4.6 нм, заключенной в барьеры $\mathrm{Hg}_{0.3} \mathrm{Cd}_{0.7} \mathrm{Te}(013)$, при $T=20 \mathrm{~K}$. Волновой вектор на рисунке направлен по [100]. Для расчета электронного спектра использовалась модель Кейна $8 \times 8$ [13].

Bсе это приводит к тому, что оже-процесс с участием 2 дырок и электрона становится намного более вероятным, чем оже-процесс с участием 2 электронов и дырки, т.е. $C_{n} \ll C_{p}$. Для дальнейших расчетов будет использовано приближение $C_{n}=0$. Отсюда следует, что для уменьшения порога генерации СИ необходимо уменьшать концентрацию неравновесных дырок. Условие инверсии населенности - разность квазиэнергий Ферми неравновесных электронов и дырок должна превышать ширину запрещенной зоны - можно обеспечить с помощью легирования донорами путем введения $\delta$-слоев, расположенных вблизи КЯ. При этом увеличение поглощения Друде будет минимальным, а уровень Ферми окажется в зоне проводимости КЯ $\mathrm{HgTe}$, и для выполнения условия инверсии населенности достаточно будет значительно меньшей концентрации неравновесных инжектируемых электронов и дырок.

\section{3. Результаты расчета}

На рис. 2 приведена зависимость пороговых плотности тока и соответствующей ей согласно формуле (2) интенсивности накачки с энергией фотона 0.54 эВ $(\lambda=2.3$ мкм), $\eta \approx 0.01$ [11] от концентрации легирующей примеси в лазере на основе $\mathrm{Hg}_{0.3} \mathrm{Cd}_{0.7} \mathrm{Te} \mathrm{c}$ квантовыми ямами $\mathrm{HgTe}$ толщиной $d=4.6 \mathrm{Hм}, Q=5$,

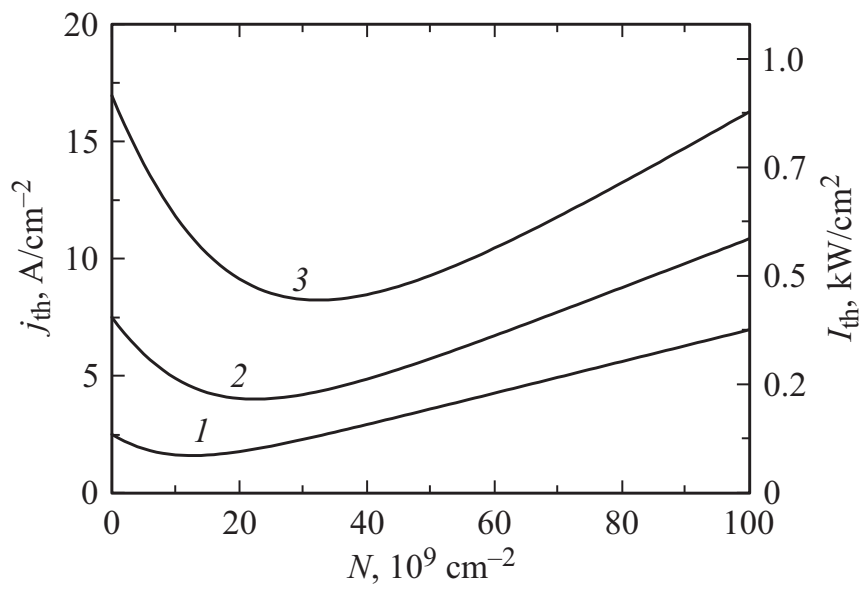

Рис. 2. Зависимости пороговых плотности тока $\left(j_{\text {th }}\right)$ и интенсивности накачки $\left(I_{\text {th }}\right)$ с $\lambda=2.3$ мкм от концентрации легирующей примеси в лазере на основе $\mathrm{Hg}_{0.3} \mathrm{Cd}_{0.7} \mathrm{Te}$ с квантовыми ямами $\mathrm{HgTe}$ толщиной $d=4.6 \mathrm{HM}, Q=5$, для трех значений температуры $T, \mathrm{~K}: 1-20,2-30,3-40$.

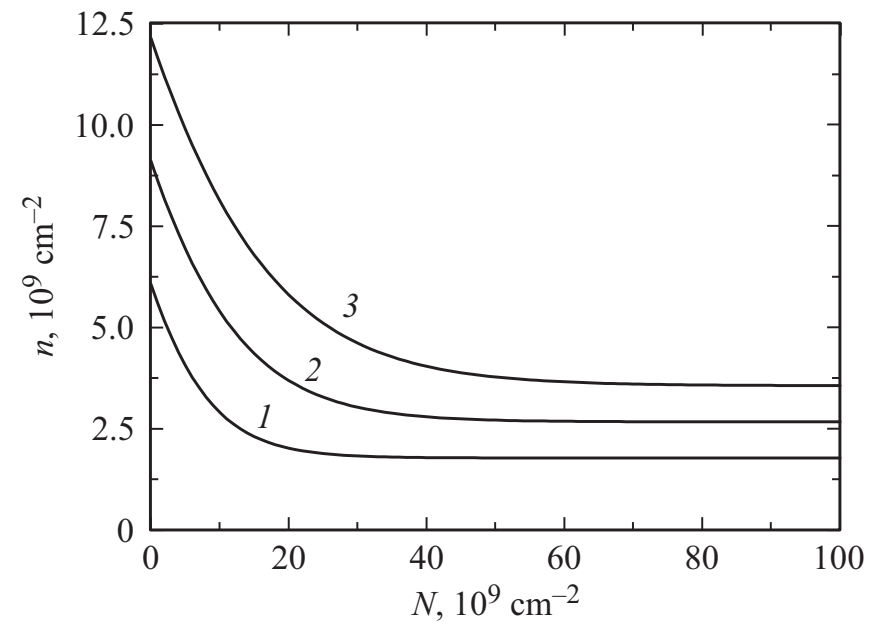

Pис. 3. Зависимости пороговой концентрации инжектированных электронов от концентрации легирующей примеси в лазеpe на основе $\mathrm{Hg}_{0.3} \mathrm{Cd}_{0.7} \mathrm{Te}$ с квантовыми ямами $\mathrm{HgTe}$ толщиной $d=4.6$ нм, $Q=5$, для трех значений температуры $T, \mathrm{~K}: 1-$ $20,2-30,3-40$.

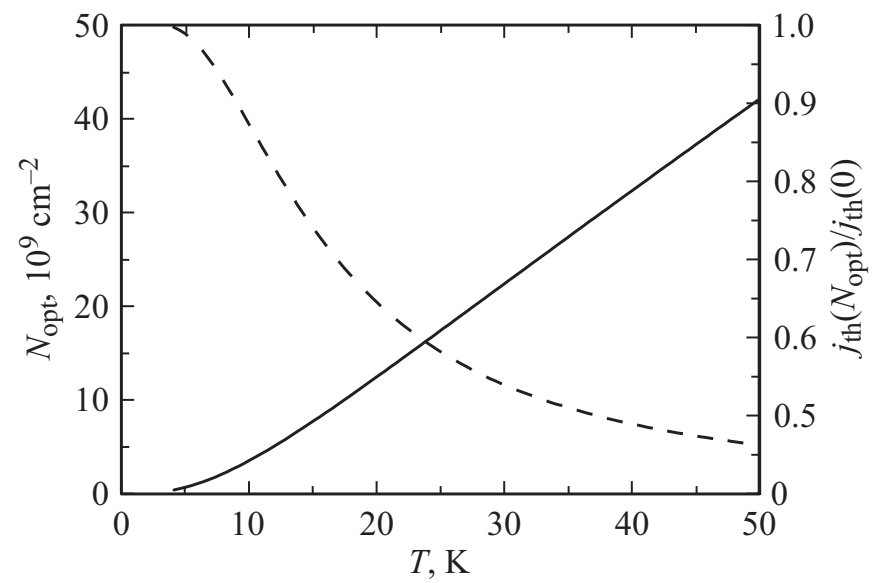

Рис. 4. Зависимости $N_{\text {орt }}$ (сплошная кривая) и отношения плотностей порогового тока для $N=N_{\text {opt }}$ и $N=0$ (штриховая кривая) от температуры.

для трех значений температуры. Так как в литературе практически отсутствуют значения параметров для оже- и излучательной рекомбинации в узкозонных КЯ $\mathrm{HgTe,} \mathrm{для} \mathrm{оценки} \mathrm{были} \mathrm{использованы} \mathrm{па-}$ раметры для объемных растворов $\mathrm{HgCdTe}$ с той же шириной запрещенной зоны: $C_{p}=1.25 \cdot 10^{-24} \mathrm{~cm}^{6} / \mathrm{c}$ и $B=1.5 \cdot 10^{-10} \mathrm{~cm}^{3} / \mathrm{c}[14]$. Время безызлучательной рекомбинации Шокли-Рида-Холла для узкозонных КЯ HgTe было определено из экспериментальных данных [15]: $\tau=1$ мкс, а масса $m=0.016 m_{0}\left(m_{0}\right.$ - масса свободного электрона) определена из аппроксимации электронного спектра на рис. 1. Характерный коэффициент потерь на выход излучения из лазера длиной 1 мм: $\alpha=10 \mathrm{cм}^{-1}$ при $\lambda \approx 20$ мкм для $T=20 \mathrm{~K}, D=5$ мкм.

Как видно из рисунка, для всех температур существует оптимальное значение концентрации полностью 
ионизованной донорной примеси $N=N_{\text {орt }}$ в $\delta$-слоях, при котором пороговые плотность тока и интенсивность накачки минимальны. Из рис. 3 видно существенное (в $\sim 3$ раза) снижение пороговых концентраций инжектируемых носителей с ростом $N$. При этом значение $N_{\text {opt }}$ практически линейно растет от $10^{9}$ до концентрации $4 \cdot 10^{10} \mathrm{~cm}^{-2}$ с ростом температуры от 4 до $50 \mathrm{~K}$, и происходит снижение порога генерации СИ более чем в 2 раза (см. рис. 4). Следовательно, можно сделать вывод, что создание в лазерных $\mathrm{HgCdTe-структурах} \mathrm{с} \mathrm{КЯ} \mathrm{HgTe}$, легированных донорной примесью $\delta$-слоев с оптимальной концентрацией, наиболее выгодно для снижения порога генерации при высоких рабочих температурах.

\section{4. Заключение}

Проведенные оценки для лазерной $\mathrm{HgCdTe-структуры}$ с КЯ НgТе, аналогичной структуре из работы [8], излучающей на $\lambda \approx 20$ мкм, показали, что при добавлении в структуру $\delta$-слоев с поверхностной концентрацией доноров $4 \cdot 10^{10} \mathrm{~cm}^{-2}$ возможно снижение порога генерации СИ более чем в 2 раза, а следовательно, возможно увеличение максимальной температуры, при которой наблюдается СИ.

Работа выполнена при финансовой поддержке гранта Российского научного фонда (проект № 17-12-01360).

\section{Список литературы}

[1] M.S. Vitiello, G. Scalari, B. Williams, P. De Natale. Opt. Express, 23 (4), 5167 (2015).

[2] V.S. Varavin, V.V. Vasiliev, S.A. Dvoretsky, N.N. Mikhailov, V.N. Ovsyuk, Y.G. Sidorov, A.O. Suslyakov, M.V. Yakushev, A.L. Aseev. Proc. SPIE, 5136, 381 (2003).

[3] S. Dvoretsky, N. Mikhailov, Yu. Sidorov, V. Shvets, S. Danilov, B. Wittman, S. Ganichev. J. Electron. Mater., 39(7), 918 (2010).

[4] N.N. Mikhailov, R.N. Smirnov, S.A. Dvoretsky, Yu.G. Sidorov, V.A. Shvets, E.V. Spesivtsev, S.V. Rykhlitski. Int. J. Nanotechnol., 3 (1), 120 (2006).

[5] S.V. Morozov, V.V. Rumyantsev, A.A. Dubinov, A.V. Antonov, A.M. Kadykov, K.E. Kudryavtsev, D.I. Kuritsin, N.N. Mikhailov, S.A. Dvoretskii, V.I. Gavrilenko. Appl. Phys. Lett., 107, 042105 (2015).

[6] S.V. Morozov, V.V. Rumyantsev, A.M. Kadykov, A.A. Dubinov, K.E. Kudryavtsev, A.V. Antonov, N.N. Mikhailov, S.A. Dvoretskii, V.I. Gavrilenko. Appl. Phys. Lett., 108, 092104 (2016).

[7] В.В. Румянцев, М.А. Фадеев, С.В. Морозов, А.А. Дубинов, К.Е. Кудрявцев, А.М. Кадыков, И.В. Тузов, С.А. Дворецкий, Н.Н. Михайлов, В.И. Гавриленко, F. Терре. ФТП, 50 (12), 1679 (2016).

[8] S.V. Morozov, V.V. Rumyantsev, M.A. Fadeev, M.S. Zholudev, K.E. Kudryavtsev, A.V. Antonov, A.M. Kadykov, A.A. Dubinov, N.N. Mikhailov, S.A. Dvoretsky, V.I. Gavrilenko. Appl. Phys. Lett., 111, 192101 (2017).
[9] В.В. Румянцев, А.М. Кадыков, М.А. Фадеев, А.А. Дубинов, В.В. Уточкин, Н.Н. Михайлов, С.А. Дворецкий, С.В. Морозов, В.И. Гавриленко. ФТП, 51 (12), 1616 (2017).

[10] А.А. Дубинов. ФТП, 50 (11), 1469 (2016).

[11] С.В. Морозов, М.С. Жолудев, А.В. Антонов, В.В. Румянцев, В.И. Гавриленко, В.Я. Алешкин, А.А. Дубинов, Н.Н. Михайлов, С.А. Дворецкий, О. Drachenko, S. Winnerl, H. Schneider, M. Helm. ФТП, 46 (11), 1388 (2012).

[12] A.A. Dubinov, V.Ya. Aleshkin. Int. J. High Speed Electron. Syst., 25, 1640018 (2016).

[13] M. Zholudev, F. Teppe, M. Orlita, C. Consejo, J. Torres, N. Dyakonova, M. Czapkiewicz, J. Wrobel, G. Grabecki, N. Mikhailov, S. Dvoretskii, A. Ikonnikov, K. Spirin, V. Aleshkin, V. Gavrilenko, W. Knap. Phys. Rev. B, 86 (20), 205420 (2012).

[14] E. Bellotti, D. D’Orsogna. IEEE J. Quant. Electron., 42 (4), 418 (2006).

[15] В.В. Румянцев, А.В. Иконников, А.В. Антонов, С.В. Морозов, М.С. Жолудев, К.Е. Спирин, В.И. Гавриленко, С.А. Дворецкий, Н.Н. Михайлов. ФТП, 47 (11), 1446 (2013).

Редактор Л.В. Шаронова

\section{Reduction of the generation threshold by doping in mid-infrared lasers based on $\mathrm{HgCdTe}$ with HgTe quantum wells}

\section{A.A. Dubinov, V.Ya. Aleshkin, S.V. Morozov}

Institute for Physics of Microstuctures, Russian Academy of Sciences, 603950 Nizhny Novgorod, Russia Lobachevsky State University of Nizhny Novgorod, 603950 Nizhny Novgorod, Russia

Abstract The possibility of a significant reduction in the interband generation threshold in mid-IR laser structures based on $\mathrm{HgCdTe}$ with $\mathrm{HgTe}$ quantum wells is proposed and analyzed. To obtain the result the structures are doped by using $\delta$-layers located near the quantum wells. It is shown that at an optimum surface donor concentration in $\delta$-layers $4 \cdot 10^{10} \mathrm{~cm}^{-2}$ and an operating temperature $>40 \mathrm{~K}$, the laser generation threshold at the wavelength of $20 \mu \mathrm{m}$ can be reduced by more than 2 times. 